\title{
Klassische Resektionsverfahren bei chronischer Pankreatitis
}

\author{
H.D. Saeger F. Dobrowolski S. Kersting D. Ockert \\ Klinik und Poliklinik für Viszeral-, Thorax- und Gefäßchirurgie, Universitätsklinikum Dresden
}

Schlüsselwörter

Chronische Pankreatitis · Duodenopankreatektomie

\section{Zusammenfassung}

Chirurgische Eingriffe werden bei $10 \%$ der Patienten mit Komplikationen der chronischen Pankreatitis erforderlich. Neben Ableitungsoperationen kommt bei fokaler Akzentuierung der Erkrankung die Resektion von Teilen der Bauchspeicheldrüse zum Einsatz. Entzündliche Tumoren des Korpus und des Schwanzbereichs werden durch linksseitige Resektion, wenn möglich Milz erhaltend, entfernt. Zu den klassischen Resektionsverfahren von Pankreaskopfprozessen gehören die Duodenopankreatektomie (DPE) nach KauschWhipple und die Pylorus erhaltende Kopfresektion (PPPD). Im eigenen Krankengut wurden von Oktober 1993 bis Mai 2001373 Patienten mit chronischer Pankreatitis behandelt. 104 Patienten wurden reseziert (27,9\%). Neben 13 Linksresektionen, davon 5 Milz erhaltend, wurden 91 DPE durchgeführt (54 Kausch-Whipple, 37 PPPD). Von den 91 Patienten, die einer DPE unterzogen wurden, hatten $25,2 \%$ der Patienten einen Diabetes mellitus. Konservativ unbeeinflussbare Schmerzen bestanden in 93\% der Fälle, ein Verschlussikterus war bei einem Drittel der Patienten aufgetreten. Der Gewichtsverlust in dieser Gruppe betrug median $14(3-30) \mathrm{kg}$. Nach der Operation traten bei 28 Patienten $(30,8 \%)$ Komplikationen auf. Fünf Patienten aus dieser Gruppe mussten reoperiert werden, keiner verstarb im postoperativen Verlauf. Für die Langzeitergebnisse konnten in einem Beobachtungszeitraum von median 20 Monaten bisher 49 Patienten nachuntersucht werden. Vier Patienten $(8,2 \%)$ waren nach einer medianen Überlebenszeit von 22 Monaten verstorben. Von den verbleibenden 45 Patienten nach DPE hatten 51,1\% im Median 7 (1-27) kg an Gewicht zugenommen. Postoperativ ist ein De-novo-Diabetes in 5 Fällen $(11,1 \%)$ aufgetreten. Dreimal $(6,1 \%)$ war nach DPE kein Diabetes mehr nachweisbar, $61,5 \%$ der Patienten wurden wieder arbeitsfähig. Wenn auch die untersuchte Patientengruppe noch klein ist, Spätergebnisse bisher nur an einem Teil der Behandelten erhoben werden konnten und der Vergleich verschiedener Serien nicht zulässig ist, scheint die klassische DPE bei der Kopfpankreatitis nach wie vor ein vertretbares Operationsverfahren zu sein.

\section{Key Words}

Chronic pancreatitis · Duodenopancreatectomy

\section{Summary \\ Classic Resection Procedures in Patients with Chronic \\ Pancreatitis}

Surgery is needed in $10 \%$ of patients with chronic pancreatitis. In cases with focal inflammation of the pancreatic head or tail, bypass procedures or partial resections are performed. If possible, the left part of the pancreas is resected, with preservation of the spleen. Duodenopancreatectomy (DPE) according to Kausch-Whipple and the pylorus-preserving resection of the pancreatic head (PPPD) belong to the classic resections. Between October 1993 and May 2001, 373 patients with chronic pancreatitis were admitted to our department. Resection was necessary in 104 patients (27.9\%). 13 left-sided resections, with splenic preservation in 5 cases, and 91 DPE were performed, 54 of them as KauschWhipple operations and 37 as PPPD. In the group of 91 DPE, $25.2 \%$ of the patients were diabetic and $93 \%$ suffered from conservatively uncontrollable pain. One third of the patients presented with obstructive jaundice and median weight loss of 14 (3-30) kg. Early postoperative complications were observed in 28 cases (30.8\%); no patient died. Up to now longterm results could be achieved in 49 patients, with a median follow-up of 20 months. Four patients $(8.2 \%)$ died after a median survival time of 22 months. $51.1 \%$ of the 45 survivors after DPE gained 7 (1-27) kg of weight. De novo diabetes occurred postoperatively in 5 patients (11.1\%). In 3 patients $(6.1 \%)$ diabetes disappeared postoperatively, $61.5 \%$ returned to work. Although this group is small, long-term results are still incomplete and the comparison of different series does not allow to draw any significant conclusions, the classic DPE for chronic pancreatitis still seems to lead to quite remarkable results.

\begin{tabular}{ll}
\hline KARGER & @ 2001 S. Karger GmbH, Freiburg \\
Fax +49 7614520714 & Accessible online at: \\
$\begin{array}{l}\text { E-mail Information@Karger.de } \\
\text { www.karger.com }\end{array}$ & www.karger.com/journals/cga
\end{tabular}

Prof. Dr. H.D. Saeger

Klinik und Poliklinik für Viszeral-, Thorax- und Gefäßchirurgie

Universitätsklinikum Carl Gustav Carus

Technische Universität Dresden

Fetscherstraße 74, D-01307 Dresden

Tel. +49 351 458-27 42, Fax -43 95 - E-mail hans-detlev.saeger@mailbox.tu-dresden.de 
Die chronische Pankreatitis ist in mehr als $80 \%$ auf einen Alkoholabusus zurückzuführen. Entsprechend wird die Langzeitprognose insgesamt ungünstig eingeschätzt. $20-50 \%$ der Patienten sterben innerhalb von 10 Jahren an den Folgen der Erkrankung und an anderen Begleitkrankheiten [1]. Eine kausale Therapie ist trotz intensiver Forschungsarbeiten bislang nicht bekannt. Die Behandlungsstrategien richten sich deshalb gegen die Komplikationen des Krankheitsbildes. Dazu gehören besonders Schmerzen sowie mechanische Abflussstörungen der Gallenwege und des Magen-Darm-Trakts. Blutungen sind selten, aber häufig lebensbedrohlich. Chirurgische Interventionen sollen zur Kontrolle dieser Komplikationen beitragen. Während der Effekt auf eine Reduzierung von Schmerzen und die Wiederherstellung mechanischer Behinderungen unbestritten ist, bleibt bisher unklar, ob die chirurgische Behandlung tatsächlich zur langfristigen Beeinflussung des Verlaufs beitragen und Spätkomplikationen der Erkrankung verhindern kann.

Ein weiteres Problem ist die Koinzidenz von chronischer Entzündung und Malignität im Pankreas [2]. Maligne Neoplasien der Bauchspeicheldrüse sind nicht selten mit entzündlich-fibrotischen Veränderungen des Organs verbunden. Ursache hierfür kann eine tumorbedingte Abflussbehinderung des Ductus Wirsungianus sein. Umgekehrt ist das Karzinomrisiko bei der chronischen Pankreatitis erhöht. Die präoperative Zuordnung einer soliden oder zystischen Raumforderung im Pankreas zu einem benignen oder malignen Prozess kann erschwert, in einigen Fällen unmöglich sein.

Entscheidend für den Erfolg der chirurgischen Therapie sind die richtige Indikationsstellung zur Operation, die Verfahrenswahl und nicht zuletzt die chirurgische Kompetenz. Das Operationsziel besteht in der Beherrschung der genannten Komplikationen unter Berücksichtigung der potentiellen Operationsrisiken und gegebenenfalls in der Kontrolle eines Krebsleidens. Besonders in der Chirurgie der gesicherten Entzündung muss soviel wie möglich von der verbliebenen Organfunktion erhalten werden. Auch daran orientiert sich die Wahl des Operationsverfahrens.

Nicht selten präsentiert sich die chronische Pankreatitis mit einer lokal akzentuierten Tumorformation. Unter den genannten Umständen ist dann die Resektion mit den besten Erfolgschancen für die Kontrolle der krankheitsbedingten Komplikationen verbunden. Die Evaluierung der eigenen Erfahrungen soll zur Einordnung des Stellenwerts der Resektion in diesen Fällen beitragen. Zu den klassischen Resektionen zählen die Duodenopankreatektomie (DPE) und die linksseitige Resektion.

\section{Patienten und Methoden}

Im Zeitraum von Oktober 1993 bis Mai 2001 wurden 1232 Patienten wegen verschiedener Pankreaserkrankungen in die chirurgische Klinik eingewiesen, 373-mal (30,3\%) war der Einweisungsgrund eine chronische Pankreatitis. Während wir in 125 Fällen (33,5\%) die weitere, konservative
Tab. 1. Komplikationen und Charakteristika bei 91 Patienten mit Kopfpankreatitis und Duodenopankreatektomie

\begin{tabular}{lll}
\hline Komplikation & \multicolumn{2}{l}{ Anzahl } \\
\cline { 2 - 3 } & $\mathrm{n}$ & $\%$ \\
\hline Schmerzen & 85 & 93,4 \\
Gallenwegstenose & 54 & 59,3 \\
$\quad$ Verschlußikterus & 30 & 33,0 \\
Duodenalstenose & 30 & 33,0 \\
Pfortaderstenose & 16 & 17,6 \\
Zystische Läsion & 38 & 41,8 \\
Diabetes mellitus & 23 & 25,3 \\
$\quad$ Insulinpflichtig & 12 & 52,2 \\
\hline Anamnesedauer (median) & & \\
$\quad$ Monate & $36(3-240)$ & \\
Gewichtsverlust (median), kg & $14(3-30)$ & \\
\hline
\end{tabular}

Therapie empfohlen haben, wurden 144 Patienten (38,6\%) durch Drainageverfahren behandelt. Insgesamt wurden 177 Patienten wegen chronischer Pankreatitis operiert (47,5\%). Die Gruppe der Resektionen beschränkte sich schließlich auf 104 Fälle (27,9\%), machte aber unter den Operierten den größten Anteil aus (104/177, 58,8\%). Diese teilten sich in 91 DPE und 13 linksseitigen Teilresektionen. Die DPE erfolgte 54-mal als Kausch-Whipple- und 37-mal als Pylorus erhaltende Operation (PPPD). Seit 1999 wurde die PPPD bevorzugt eingesetzt. Das linksseitige Pankreas wurde nicht mit dem Ziel der Schmerzkontrolle durch subtotale Entfernung, sondern zur Beseitigung von zystischen oder soliden Raumforderungen in Korpus oder Schwanz reseziert.

In der Resektionsgruppe waren 55 solide (48,2\%) und 49 zystische «Tumoren» $(43 \%)$ Indikationen zum chirurgischen Eingriff. Daneben wurden bei inflammatorischen, zystischen Prozessen Ableitungsoperationen und interventionelle Eingriffe vorgenommen. In einer ausgewählten Gruppe von 21 Pankreaszysten und -pseudozysten wurde retrospektiv die Indikation zur Bypassoperation oder zur Resektion untersucht. Die Resektion kam bei länger bestehender Symptomatik (52 vs. 17,7 Monate), kleineren Zysten (Durchmesser 5,7 vs. 7,3 cm) und bei älteren Patienten (45,1 vs. 39,1 Jahre) zum Einsatz.

Komplikationen und Charakteristika der Patienten mit Kopfresektionen $(\mathrm{n}=91)$ sind in Tabelle 1 aufgeführt.

\section{Operationstechnik}

Bei der linksseitigen partiellen Resektion des Organs $(\mathrm{n}=13)$ wurde, wenn möglich, die Milz einschließlich ihrer Arterie und Vene erhalten (5/13). Alle mit dem Pankreas kommunizierenden Äste der Arteria und Vena lienalis wurden zwischen Ligaturen oder Durchstechungsligaturen durchtrennt. Bei freiem Abfluss des Pankreasgangs über die Papilla Vateri wurde der Duktus in der Resektionsebene mit einer Durchstechungsligatur versorgt. Darüber erfolgte die Übernähung des Parenchyms mit Einzelknopf- oder 8er Nähten. Das Nahtmaterial bestand immer aus 3-0 resorbierbaren, geflochtenen PGS-Fäden. Bei verschlossenem Gang oder unklaren Verhältnissen wurde die Resektionsfläche in Pankraskorpus oder -kopf mit einer nach Roux ausgeschalteten Jejunumschlinge anastomosiert (4/13). Grundsätzlich wurde die entzündliche Läsion so sparsam wie möglich reseziert, um soviel Parenchym des Pankreas wie möglich zu erhalten.

Das klassische Resektionsverfahren bei chronischer Kopfpankreatitis besteht in der DPE. Diese Operation nach Kausch-Whipple ist mit der Entfernung von Pankreaskopf, Duodenum, Gallenblase und Ductus choledochus sowie der (Teil-)Resektion des Magenantrums verbunden. Bei der 
Tab. 2. Stationäre Wiederaufnahme und Reoperationen im Langzeitverlauf nach DPE bei chronischer Pankreatitis $(\mathrm{n}=91)$

\begin{tabular}{lcl}
\hline Indikation & Anzahl & Reoperation \\
\hline Schmerzen & 2 & 0 \\
Abszess, Restpankreatitis & 6 & 2 \\
Blutung & 1 & 1 \\
Ileus & 4 & 2 \\
Peptisches Ulkus & 2 & 0 \\
\cline { 2 - 3 } Patienten gesamt & 10 & 5 \\
\hline
\end{tabular}

PPPD bleibt der Magen einschließlich einer kurzen, proximalen Duodenalmanschette erhalten. Die Rekonstruktion wurde mit einer nach Roux ausgeschalteten, meist hinter der Mesenterialwurzel in den Oberbauch transponierten Jejunumschlinge durchgeführt. Der gesamte Querschnitt des Restpankreas, der Ductus hepaticus communis und das Duodenum wurden in Abständen von jeweils etwa $8 \mathrm{~cm}$ mit der Dünndarmschlinge anastomosiert.

\section{Ergebnisse}

Die folgenden Daten beziehen sich auf 91 Patienten, bei denen wegen chronisch entzündlicher Veränderungen der Pankreaskopf reseziert wurde. Der postoperative Krankenhausaufenthalt betrug median 14 (9-91) Tage, die Überwachung auf der Intensivstation 4 (2-62) Tage. Bei 28 Patienten $(30,8 \%)$ kam es $\mathrm{zu}$ postoperativen Komplikationen, von denen in 5 Fällen eine Reoperation erforderlich wurde. Dabei handelte es sich 1-mal um einen postoperativen mechanischen Ileus und 4-mal um intraabdominale Blutungen. Alle Komplikationen konnten erfolgreich kontrolliert werden. Kein Patient verstarb während des Klinikaufenthalts.

Zur Erhebung der Langzeitergebnisse konnten bisher $49 \mathrm{~Pa}-$ tienten nach DPE nachuntersucht werden. Die Nachbeobachtungszeit betrug median 20 (5-59) Monate. Von diesen verstarben 4 Patienten (8,2\%). Die Todesursachen bestanden in einer Restpankreatitis mit Ausbildung eines pankreatogenen Aszites und Sepsis, einem rupturierten Milzarterienaneurysma, einer Lungenembolie und einem Myokardinfarkt. Die Überlebensdauer der Verstorbenen betrug median 22 Monate.

Bei 34 der verbleibenden 45 Patienten bestanden keine oder nur geringe Schmerzen (75,6\%). Der Alkoholabusus sank von initial $80 \%$ postoperativ auf $37,8 \%$. Fünf Patienten $(11,1 \%)$ entwickelten postoperativ einen De-novo-Diabetes mellitus, davon waren 4 insulinpflichtig. In 3 Fällen war ein präoperativ bestehender Diabetes mellitus postoperativ nicht mehr nachweisbar. Die Einstellung auf orale Antidiabetika konnte postoperativ abgesetzt werden. In $51,1 \%$ der Fälle kam es postoperativ zu einer Gewichtszunahme, die median 7 (1-27) kg betrug. Von 26 Patienten, die präoperativ arbeitsfähig waren, nahmen 16 postoperativ die Arbeit wieder auf (61,5\%).
Zehn von 91 Patienten wurden im Langzeitverlauf erneut stationär aufgenommen, davon mussten 5 aus verschiedenen Gründen operiert werden. Die Gründe für die Wiederaufnahmen und Operationen sind in Tabelle 2 aufgelistet.

\section{Diskussion}

Die chronische Pankreatitis ist nach wie vor in vieler Hinsicht ein besonderes Problem. Während die Ätiologie in mehr als $80 \%$ auf Alkoholismus zurückzuführen ist, verbleiben etwa $10 \%$ völlig unklare Ursachen für die Entstehung. Wiederum $10 \%$ müssen wegen Komplikationen der Erkrankung operiert werden [1]. Ziele dabei sind die Beseitigung unkontrollierbarer Schmerzen und mechanischer Hindernisse unter Erhaltung einer möglichst großen Menge von Pankreasgewebe. Unter der früheren Vorstellung der Entwicklung einer chronischen Pankreatitis durch Drucksteigerung im Pankreasgangsystem schien die Entlastung des Ductus pancreaticus durch Ableitungsoperationen die Methode der Wahl zu sein. Nur selten besteht allerdings die unter dieser Vorstellung zu erwartende duktale Dilatation. Die heute geforderte Weite von wenigstens $7-8 \mathrm{~mm}$ haben wir nur selten beobachtet. In Europa sind wir vielmehr - offenbar anders als in den USA - häufig mit einer umschriebenen Kopfpankreatitis konfrontiert. Die dann auftretenden Schmerzen durch Gewebedruck und Untergang von Nervenscheiden, verbunden mit einer Abflussbehinderung der Gallenwege und des Ductus Wirsungianus sowie einer möglichen Pfortader- und/oder Duodenalstenose werden am konsequentesten durch die Resektion oder Teilentfernung des Pankreaskopfes kontrolliert.

Mit zunehmender Erfahrung mit Resektionen in der Pankreaschirurgie konnten die Raten der Operationsletalität auf $1-3 \%$ gesenkt werden [3-8]. Immer wieder wurde $\mathrm{zu}$ Recht postuliert, dass eine niedrige Komplikations- und Letalitätsrate Voraussetzung dafür ist, bei dem benignen Leiden die technisch aufwendigen und mit potentiell hohem Risiko verbundenen Resektionen am Pankreas zu vertreten. Obwohl die Patienten nach lange andauernder Erkrankung durch Gewichtsverlust, Analgetikabedarf, nicht selten fortgesetzten Alkoholismus und damit reduzierten Immunstatus in schlechtem Allgemeinzustand sind, ist das Operationsrisiko bei der chronischen Pankreatitis geringer als beim Karzinom.

Für den langfristigen Therapieerfolg ist bereits die Indikationsstellung zur Resektion und damit die Selektion der Patienten von wesentlicher Bedeutung. Leidensdruck, anhaltend mechanische Abflussbehinderungen, z.B. des biliären Systems, aber auch die Compliance der Patienten fließen in den Entscheidungsprozess ein. Im eigenen Krankengut betrugen die Dauer der Schmerzanamnese median 36 Monate und der mediane Gewichtsverlust $14 \mathrm{~kg}$. Die Anamnesedauer vor Entscheidung zur chirurgischen Therapie entspricht der anderer Serien [3]. Nur 27,9\% der 373 eingewiesenen Patienten wurden reseziert. Daneben ist der Malignomverdacht bei um- 
schriebenen Vergrößerungen im Pankreas von Bedeutung. Im eigenen Krankengut wurden 47,3\% der Patienten mit entzündlichen Pankreaskopfvergrösserungen unter dem Verdacht eines malignen Tumors eingewiesen. Mit der präoperativen Diagnostik lässt sich dieser Anteil in der Regel auf 5-10\% senken. Selbst unter Einsatz aller laborchemischen und bildgebenden Verfahren inklusive der Positronenemissionstomografie ist prä- und selbst intraoperativ eine definitive Zuordnung nicht immer möglich. Erst das resezierte Präparat lässt eine (relativ) sichere Einordnung zu [2, 5].

Das klassische Resektionsverfahren bei der chronischen Kopfpankreatitis ist die DPE nach Kausch-Whipple. A.O. Whipple publizierte die erste größere Serie bei chronischer Pankreatitis [9]. In den folgenden Jahrzehnten hat sich dieses Verfahren weltweit mehr und mehr durchgesetzt. Die Ergebnisse wurden kontinuierlich verbessert. Die Pylorus erhaltende DPE wurde von Traverso und Longmire vorgestellt und hat sich inzwischen weltweit etabliert $[4,6,10]$. Dennoch wurden bereits in den 70er Jahren neue Operationsmethoden entwickelt. Unter dem Eindruck einer Übertherapie mit der DPE nach Kausch-Whipple entwickelte Beger die Duodenum erhaltende Pankreaskopfresektion (DPPHR) [11], Frey reduzierte die Kopfresektion auf eine partielle Gewebeexzision [12] und Prinz propagierte die Drainageoperation des Gangsystems [13]. Die totale DPE ebenso wie die von Russell entwickelte Duodenum erhaltende totale Pankreatektomie [14] sind heute bei benigner Indikation nahezu vollständig verlassen. Ebenso spielt die unter der Vorstellung der optimalen Schmerzbeseitigung durchgeführte subtotale linksseitige Pankreasresektion bei der chronischen Pankreatitis keine Rolle mehr.

Wie ist heute der Stellenwert der verschiedenen Resektionsverfahren bei der chronischen Pankreatitis einzuordnen? Die linksseitige Resektion kommt in der Regel nur bei der seltenen, umschriebenen Schwanzpankreatitis zum Einsatz. Hier liegen überwiegend - im eigenen Krankengut waren es 11 von 13 - zystische Läsionen vor, die möglichst Milz erhaltend unter Schonung der Arteria und Vena lienalis entfernt werden [15, 16]. Die heute konkurrierenden Verfahren bei der Kopfpankreatitis sind die DPE, die DPPHR und die Operation nach Frey. Darunter ist die DPPHR die am besten dokumentierte Methode. Beger hat die Früh- und Spätergebnisse seiner Serie mit 507 Patienten im Zeitraum von 1972-1998 eingehend analysiert [3]. Der postoperative stationäre Aufenthalt betrug median 14,5 (7-87) Tage. 28 Patienten (5,6\%) benötigten eine Relaparotomie, und die Operationsletalität betrug 0,8\%. Diese Daten entsprechen den Ergebnissen der eigenen, noch kleinen Serie mit 14 (9-91) Tagen und 5,5\% Relaparotomien. Eine Operationsletalität nach DPE trat bisher nicht auf.

Der Vorteil der Duodenum erhaltenden Verfahren wird besonders für die mittel- bis langfristigen Ergebnisse beschrieben. Tatsächlich ist die erreichte Schmerzfreiheit mit 91,3\% in der Analyse von Beger den eigenen Erfahrungen mit nur 75\% deutlich überlegen. Die Erklärung dafür bleibt unklar, bedeutet doch die DPE eine vollständigere Entfernung des Pankre- askopfgewebes. Für die Analyse der Spätergebnisse konnten wir bisher nur eine kleine Gruppe von 49 Patienten (53,8\%) evaluieren. Die Spätletalität bei einer Nachbeobachtungszeit von 20 (5-59) Monaten betrug 8,2\% (5/49) bei einer medianen Überlebenszeit der Verstorbenen von 22 Monaten. Nach einem medianen Follow-up von 5,7 Jahren nach DPPHR lag die Spätletalität bei 12,6\% (49/338). Unter Beachtung der Todesursachen ist allerdings fraglich, ob eine der gebräuchlichen Operationsmethoden einen begünstigenden Einfluss auf die Spätletalität der chronischen Pankreatitis haben kann.

Die Erhaltung der Duodenalpassage (enteroinsulinäre Achse) soll insbesondere die langfristigen funktionellen Vorteile begründen. Präoperativ bestand ein Diabetes mellitus in der Gruppe der DPPHR-Patienten bei $25 \%$, in der eigenen Patientengruppe bei $25,3 \%$ der Betroffenen. Ein postoperativer insulinpflichtiger De-novo-Diabetes trat in der ersten Gruppe bei $21 \%$ (bezogen auf alle Patienten, 64/303), nach DPE bei $13,8 \%$ der Patienten (bezogen auf die Gruppe der präoperativen Nichtdiabetiker, 19/45) auf. Eine Verbesserung des diabetischen Status wurde nach DPPHR in 11\% (34/303), nach DPE in 6,7\% der Fälle (3/45 Nachuntersuchungen) beobachtet. Die stationäre Wiederaufnahme war nach DPE bei $11 \%$, nach DPPHR bei $12,5 \%$ der Patienten erforderlich. Die Rückkehr zur Arbeit war entsprechend bei 61,5\% (DPE) bzw. bei 69\% (DPPHR) der Betroffenen möglich. Bezogen auf das präoperative Ausgangsgewicht nahmen 51\% der nachuntersuchten Patienten nach DPE median 7 kg an Gewicht zu. Präoperativ bestand bei $80 \%$ der eigenen Patienten ein Alkoholabusus, postoperativ lag die Rate bei $37,8 \%$. Vor und nach DPPHR lagen die entsprechenden Werte bei 81 und $41 \%$.

Wenngleich der Vergleich derart unterschiedlicher Serien keine allgemeingültigen Rückschlüsse erlaubt, so ist die Kongruenz der Daten in vieler Hinsicht doch erstaunlich. Die eigene Patientengruppe ist bisher klein, die Nachbeobachtungsperiode kurz und insbesondere die Anzahl der nachuntersuchten Patienten unvollständig. Wahrscheinlich ist, dass mit der Beobachtungszeit die Entwicklung eines Diabetes, die Spätletalität und die Anzahl der stationären Wiederaufnahmen zunehmen werden. Es wird schwer sein, die nahezu lückenlose Dokumentation des eigenen Patientenguts zu erreichen, wie dies Beger und seinen Schülern beispielhaft gelungen ist. Inzwischen liegen prospektiv randomisierte Studien zum Vergleich der Duodenum erhaltenden Methode mit der DPE vor. Die Vorteile der DPPHR versus Kausch-Whipple-Operation [17] bzw. der DPPHR versus PPPD [18] wurden beschrieben. Eine kürzlich publizierte prospektiv randomisierte Studie hat in einer vergleichenden Untersuchung den Wert einer erweiterten Frey-Operation gegenüber der Pylorus erhaltenden DPE dargestellt. Hier wurde ein Vorteil der Operation nach Frey besonders im Hinblick auf die Lebensqualität beobachtet [5]. Andere Autoren haben auch nach DPE eine nahezu normale Lebensqualität beschrieben [19]. Dabei ist der Vergleich Whipple versus PPPD offenbar ohne wesentliche Unterschiede [6]. 
In erfahrenen Zentren sind die verschiedenen Methoden der Pankreaskopfresektion mit Ergebnissen verbunden, die auf die chronische Pankreatitis gegenüber dem Spontanverlauf offenbar einen günstigen Einfluss haben. Die klassischen Resektionsverfahren, und hier besonders die PPPD, sind in aktuellen Untersuchungen auch bezüglich der längerfristigen funktionellen Befunde gegenüber früheren Ergebnissen mit der Kausch-Whipple-Operation eindeutig verbessert. Die Duodenalpassage ist bei der DPPHR und der Frey-Operation für das Pankreassekret nicht zu erhalten. In den Fällen, die eine zusätzliche Dekompression der Gallenwege benötigen, fließen Galle und Pankreassekret in eine nach Roux ausgeschaltete Dünndarmschlinge. In der von Beger publizierten Serie war dies in $24 \%$ der Operationen erforderlich. Inwieweit hier das in seiner Kontinuität erhaltene, aber wenigstens partiell denervierte Duodenum in der ursprünglichen Funktion wirksam werden kann, ist fraglich.

Bleibt schließlich der - wenn auch kleine - Anteil des ungeklärten Karzinomverdachts. In diesen Fällen sollte primär die DPE zur Anwendung kommen. Unter Berücksichtigung der derzeitigen Erkenntnisse und der noch offenen Fragen haben neben den Duodenum erhaltenden auch die klassischen Resektionsverfahren durchaus einen Stellenwert im differentialtherapeutischen Spektrum chirurgischer Resektionen bei der chronischen Pankreatitis.

\section{Literatur}

1 Buehler L, Schmidlin F, de Perrot M, Borst F, Mentha G, Morel Ph: Long-term results after surgical management of chronic pancreatitis. Hepatogastroenterology 1999;46:1986-1989.

2 Saeger HD, Schwall G, Trede M: Die partielle Duodenopankreatektomie nach Whipple - Stellenwert in der Behandlung der chronischen Pankreatitis. Zentralbl Chir 1995;120:287-291.

3 Beger HG, Schlosser W, Friess HM, Büchler MW: Duodenum-preserving head resection in chronic pancreatitis changes the natural course of the disease. A single-center 26-year experience. Ann Surg 1999;230:512-523.

4 Braasch JW, Rossi RL: Partial pancreatoduodenectomy for chronic pancreatitis; in Trede M, Carter DC (eds): Surgery of the Pancreas. New York, Churchill Livingstone, 1997, pp 357-367.

5 Izbicki JR, Bloechle C, Broering DC, Knoefel WT, Kuechler T, Broelsch CE: Extended drainage versus resection in surgery for chronic pancreatitis. A prospective randomized trial comparing the longitudinal pancreaticojejunostomy combined with local pancreatic head excision with the pylorus-preserving pancreatoduodenectomy. Ann Surg 1998: 228:771-779.

6 Jimenez RE, Fernandez-del-Castillo C, Rattner DW, Chang YC, Warshaw AL: Outcome of pancreaticoduodenectomy with pylorus preservation or with antrectomy in the treatment of chronic pancreatitis. Ann Surg 2000;231:293-300.
7 Trede M, Schwall G, Saeger HD: Survival after pancreaticoduodenectomy: 118 consecutive resections without an operative mortality. Ann Surg 1990;221:447-458.

8 Yeo CJ, Cameron JL, Sohn TA, et al: Six hundred fifty consecutive pancreaticoduodenectomies in the 1990s: Pathology, complications, outcomes. Ann Surg 1997;226:248-260.

9 Whipple AO: Radical surgery for certain cases of pancreatic fibrosis associated with calcerous deposits. Ann Surg 1946;124:991-1006.

10 Traverso LW, Longmire WP: Preservation of the pylorus in pancreaticoduodenectomy. Surg Gynecol Obstet 1978;146:959-962.

11 Beger HG, Witte C, Kraas E, Bittner R: Erfahrung mit einer das Duodenum erhaltenden Pankraskopfresektion bei chronischer Pankreatitis. Chirurg 1980;51:303-309.

12 Frey CF, Smith JG: Description and rationale of a new operation for chronic pancreatitis. Pancreas 1987;2:701-707.

13 Prinz RA, Greenlee HB: Pancreatic duct drainage in 100 patients with chronic pancreatitis. Ann Surg 1981;194:313-320.

14 Russell RCG: Total pancreatoduodenectomy for chronic pancreatitis; in Trede M, Carter DC (eds): Surgery of the Pancreas. New York, Churchill Livingstone, 1997, pp 369-378.
15 Lillemoe KD, Kaushal S, Cameron JL, Sohn TA, Pitt HA, Yeo CJ: Distal pancreatectomy: Indications and outcomes in 235 patients. Ann Surg 1999; 229:693-700.

16 Schlosser W, Rueck B, Schoenberg MH, Beger HG Pankreaslinksresektion bei chronischer Pankreatitis - mit oder ohne Splenektomie ? Langenbecks Arch Chir Kongressbericht 1997;suppl II:1084 1086.

17 Klempa I, Spatny M, Menzel J, Baca I, Nustede R, Stöckmann F, Arnold W: Pankreasfunktion und Lebensqualität nach Pankreaskopfresektion bei der chronischen Pankreatitis. Chirurg 1995;66:350-359.

18 Buechler MW, Friess H, Müller MM, Beger HG: Randomized trial of duodenum preserving pancreatic head resection versus pylorus-preserving Whipple in chronic pancreatitis. Am J Surg 1995; 169:65-70.

19 Huang JJ, Yeo CJ, Sohn TA, Lillemoe KD, Sauter PK, Coleman JA, Hruban RH, Cameron JL: Quality of life and outcomes after pancreaticoduodenectomy. Ann Surg 2000;231:890-898. 